DAVID L. WEBSTER.

\title{
NOTES ON PAGE'S THEORY OF HEAT RADIATION.
}

By David L. Webster.

$\mathrm{T}^{\mathrm{H}}$

HE paper referred to in the above title was published by Page in the Physical Review of February, I9I6, obtaining Planck's radiation formula by a method consistent with the classical electrodynamics and not involving so many radical assumptions as Planck's theory. Having made a similar attempt myself in $1915,{ }^{1} \mathrm{I}$ am interested to see that Page and I were led independently to what are essentially the same fundamental assumptions. Nevertheless there are certain differences that seem to make my theory more consistent with phenomena. Moreover, while Page's theory seems to involve fewer assumptions, it appears on closer examination to imply one that looks less reasonable than those it eliminates.

The fundamental assumptions common to both theories are (I) that the connection between electricity and the ether is that of the classical electrodynamics, (2) that the oscillator contains a rotational degree of freedom storing energy in a non-radiating form, and (3) that under the proper conditions energy can be transferred by an internal mechanism between the radiating and non-radiating degrees of freedom.

In my theory this oscillator is an electron, assumed to have the properties ascribed to electrons by Parson in his "Magneton Theory of the Structure of the Atom," 2 developed for the explanation of chemical and magnetic phenomena. According to Parson the electron, or magneton, as he calls it, is a very thin ring of negative electricity something like $\mathrm{I} .5 \times \mathrm{IO}^{-9} \mathrm{~cm}$. in radius, revolving with a velocity of the order of that of light, and therefore having magnetic, as well as electrostatic, properties.

The magneton thus conceived as a continuous ring rotating in its own plane has energy stored in a non-radiating form. If the electricity is movable on the ring in any other way than as a rigid mass, the alternating external force of a light wave will induce oscillations on it capable of absorption and radiation of energy. These induced oscillations were shown in my paper to give an explanation of refraction, diffraction and allied phenomena almost exactly like that of the classical electron theory. At the same time the internal mechanism assumed above for

1 Amer. Acad. Proc., Jan., I915.

${ }^{2}$ Smithsonian Miscellaneous Collections, Volume 65, No. II, I9I5. 
Vol. VIII.] NOTES ON PAGE'S THEORY OF HEAT RADIATION.
No. I.

transferring energy between the oscillations and the circulation can be supposed to damp the induced oscillations, transferring their energy to the rotation, until the energy accumulated above the initial value reaches some definite multiple of the quantum. At such a point it is assumed that the process may be reversed, starting a larger oscillation, which will be maintained constant at the expense of the circulation, until the excess energy is all radiated away. The probability, $\eta$, that such an oscillation will start when the stored energy reaches a given multiple of $h \nu$ is given by Planck's condition $(\mathrm{I}-\eta) / \eta=p I$, where $I$ is the mean square of the electric field per unit frequency interval, and $p$ is determined so as to give the Jeans-Rayleigh law at low frequencies. The entropy of the system is then found by Planck's equation $S=k \log W$, where the microscopic state of a system is determined by the total accumulated energy of each oscillator. The derivation of Planck's law in this theory is therefore almost exactly like his. The advantage of this system is that, unlike his, it is consistent not only with phenomena of heat radiation but with ordinary optical phenomena as well and with limitations of amplitude imposed by known sizes of atoms, and that besides correlating the phenomena of heat radiation and optics, it connects them also with those of magnetism, atomic volume relations, and chemical affinities.

In Page's theory the exact nature of the oscillator is not specified, but it is suggested that it might be a rotating electron or group of electrons. Nothing stated there would prevent one from changing that assumption to make it the magneton. The first radical difference between the two theories is that in Page's the oscillations are assumed to be stable only if their energy, without regard to that of the rotation, is very close to an integral multiple of $h \nu$. This assumption involves two serious difficulties. First, as I have pointed out in connection with Planck's theory, such a vibration involves amplitudes that are apparently too large for the atom; and second, it is not evident how such a restriction to definite energies of vibration can agree with the optical phenomena explained by the ordinary dispersion theory. Since the opportunity to correlate heat radiation with these optical phenomena, as my theory does, seems to be really the chief object to be gained by combining the classical electrodynamics and the quantum hypothesis, this point appears important in deciding between the two theories.

With regard to the internal mechanism of transfer, Page assumes that it acts in such a way as to neutralize any difference between the rates of radiation and absorption by the oscillations, keeping them constant except in collisions and placing the gain or loss in the rotation. Then he calculates the entropy of the system from Planck's postulate $S=k \log W$, 
determining the microscopic state by the energy of each oscillation, without regard to what is stored in the corresponding rotation. Thus this theory appears to gain in simplicity by eliminating the assumption $(\mathrm{I}-\eta) / \eta=p I$ made in Planck's theory and mine.

There is, however, an important difference between the way this equation $S=k \log W$ is used in Page's theory and in the others. For as Planck has pointed out, ${ }^{1}$ this postulate cannot be true unless all possible microscopic states are equally probable, since otherwise the most probable macroscopic state will not be the one with the largest value of $W$, and the entropy thus defined will not be a maximum. In the case of Planck's theory and mine, the postulate is justifiable by a proof that this equality of probabilities is a direct result of the previous assumptions, or by the proof given by Planck that the same distribution results from this postulate as from the previous assumptions. In Page's theory, on the other hand, the equality of probabilities is really a new assumption implied in the postulate $S=k \log W$.

The question now is, Is this assumption more or less reasonable than Planck's $(\mathbf{I}-\eta) / \eta=p I$. If the oscillators were independent conservative systems, exchanging energy only by quanta, the implied assumption would amount only to the condition that each one could gain or lose a quantum with a readiness independent of the number already in its possession, so long as it did not have to go below zero. But since the oscillators are not conservative, there will always be some, radiating faster than they absorb, that must stop oscillating for lack of a supply of stored energy. Consequently others, absorbing faster than they radiate, must be started into larger vibrations. This introduces the necessity for some sort of a law of starting oscillations from the stored energy. This law might take a form somewhat like the repetition of the condition $(\mathrm{I}-\eta) / \eta=p I$ on passing each multiple of the quantum in the storage system.

Now this condition, as applied by Planck, seems extremely plausible. For one might readily suppose the probability of radiation, $\eta$, if small, to be proportional to the time required for the stored energy to get through the neighborhood of the value $n h \nu$, that is, inversely to $I$. On the other hand if the probability, $\mathrm{I}-\eta$, of getting through, is small, one might assume it proportional to the speed of acquiring energy, and therefore to $I$. The simplest way to combine these assumptions is Planck's $(\mathrm{I}-\eta) / \eta=p I$. One might suppose it would be possible to apply this equation, in some form, to the assumption implied in Page's theory, but

${ }^{1}$ Heat Radiation, translation by Masius, I914, p. I25. 
Vol. VIII.] NOTES ON PAGE'S THEORY OF HEAT RADIATION.
No. r.

an attempt which I have made to do so led to a value of $\eta$ dependent on $n$, and I do not at present see any way to avoid this, although there may be one.

The net result, then, is that Page's theory shows an apparent inconsistency with data on atomic magnitudes, does not cover such a large range of phenomena as mine, and involves at least as many radical assumptions, if not more.

Jefferson Physical Laboratory,

Cambridge, Mass. 